\title{
Influencia de los rasgos de personalidad en la elección de la técnica de diálisis
}

\author{
Dolores Marquina Parra \\ Conchi Blasco Cabaña \\ Nuria Mañé Buixo \\ Esther Ponz Clemente \\ Joan Carlos Martínez Ocaña \\ Elios Yuste Jiménez \\ Manuel García García
}

Servicio de Nefrología

Corporación Parc Taulí

Sabadell (Barcelona)

\section{RESUMEN}

La Insuficiencia Renal Crónica se acompaña de problemas físicos y una fuerte dosis de ansiedad. Creemos que los rasgos de personalidad y el soporte psicosocial podrían influir en la elección de la técnica de diálisis.

Nuestro objetivo es valorar como influyen los rasgos de personalidad en la elección de la técnica de diálisis. Desde 1996 disponemos, en el Servicio de $\mathrm{Ne}^{-}$ frología, de un registro prospectivo que recoge variables relacionadas con la elección de la técnica de diálisis.

En el año 2003 realizamos un estudio transversal en todos los enfermos estables en diálisis de nuestro centro, sobre los Rasgos del Carácter, se administraron los cuestionarios:

- Cuestionario de personalidad de Eysenk (EPQ RS)

- Escala de adaptación social (PSA)

- Test de afrontamiento (COPE)

Se han estudiado 39 pacientes de los cuales 20 optaron por hemodiálisis y 19 por diálisis peritoneal.

No hemos hallado diferencias en cuanto a las variables sociales ni tampoco en el test de personalidad,

Correspondencia:

Dolores Marquina

Servicio de Nefrología

Corporació Parc Taulí S/n

08207 - SABADELL solamente se ha hallado una diferencia estadísticamente significativa en la escala desadaptativa del test de COPE. Se observa que los pacientes que eligen DP muestran una mayor capacidad para afrontar los problemas, no tenían mejor soporte y adaptación social, ni una personalidad menos neurótica.

$\begin{array}{ll}\text { PALABRAS CLAVE: } & \text { DIÁLISIS PERITONEAL } \\ & \text { AFRONTAMIENTO } \\ & \text { PERSONALIDAD } \\ & \text { ELECCIÓN }\end{array}$

\section{THE INFLUENCE OF PERSONALITY TRAITS IN THE CHOICE OF DIALYSIS TECHNIQUE}

\section{SUMMARY}

Chronic Renal Insufficiency is accompanied by physical problems and a strong dose of anxiety. We believe that the personality traits and psychosocial support might influence in the choice of dialysis technique.

Our objective is to evaluate how personality traits influence the choice of dialysis technique. In the Nephrology Service, since 1996 we have had a prospective register that gathers variables related to the choice of dialysis technique.

In 2003, we performed a transversal study on all stable dialysis patients in our unit of the Character Traits, with the following questionnaires:

- Eysenk personality questionnaire (EPQ RS)

- Social adaptation scale (PSA)

- Confronting test (COPE) 
Thirty-nine patients were studied, of which 20 opted for haemodialysis and 19 for peritoneal dialysis.

We found no differences with respect to the social variables nor in the personality test, only one statistically significant difference was found on the deadaptive scale of the COPE test. It was observed that the patients choosing PD showed a greater capacity to face problems, did not have better support and social adaptation or a less neurotic personality.

KEYWORDS: PERITONEAL DIALYSIS

CONFRONTATION

PERSONALITY

CHOICE

\section{INTRODUCCIÓN}

La llegada a la situación de insuficiencia renal crónica (IRC) terminal se acompaña de importantes problemas físicos y una fuerte dosis de ansiedad. No hay duda de que se trata de una situación vital y estresante, de manera que los rasgos de personalidad del paciente y el soporte psicosocial de que disponga podrían influir en la elección de la técnica de diálisis.

El tipo de carácter y, en general, la forma de enfrentarse a los problemas son características individuales que no parecen modificarse a lo largo del tiempo y que se pueden valorar a través de un test de personalidad, un test de adaptación social y de un test de afrontamiento de los problemas de la vida diaria.

Apenas hemos encontrado bibliografía al respecto que analice si estos rasgos de personalidad influyen en la elección de la técnica de diálisis ${ }^{(1)}$. Nuestra hipótesis es que los enfermos con personalidades más neuróticas, con mayores dificultades para afrontar problemas y menor soporte psicosocial escogerían con menor frecuencia la diálisis peritoneal como primera opción dialítica.

\section{OBJETIVO}

Valorar cómo influyen los rasgos de personalidad en la elección de la técnica de diálisis.

\section{MATERIAL Y MÉTODOS}

Desde 1996 disponemos, en el Servicio de Nefrología de la Corporación Parc Taulí, de un registro prospectivo que recoge una serie de variables sobre la elección de la técnica de diálisis (Programa de Ayuda a la Decisión [PAD] $)^{(2)}$. Por otro lado, entre 2002 y 2003 realizamos un estudio transversal en todos los enfermos crónicos en diálisis de nuestro centro para valorar la influencia de los factores psicosociales en la calidad de vida, después de excluir todos aquellos enfermos con demencia (puntuación del Minimental Examination Test inferior a 23).

Tras solicitar y obtener el consentimiento informado, se incluyeron 75 enfermos en el estudio, pero sólo disponemos de datos completos del PAD de 39 enfermos, que son los pacientes analizados en este trabajo. Los enfermos que entraron en el programa de diálisis crónica procedentes de otros centros, del trasplante renal o de la unidad de agudos, sin un control nefrológico previo, no suelen recibir la información sobre las técnicas de diálisis de manera estructurada a través del PAD y, por tanto, no son analizados en este trabajo.

Las variables recogidas en el momento de la información sobre las técnicas de diálisis (PAD) fueron:

- Demográficas: edad, sexo, estado civil, lugar de residencia.

- Soporte social: tipo de convivencia, cuidador principal.

- Situación socioeconómica. Situación laboral.

- Nivel de estudios.

- Etiología de la IRC.

- Problemas de visión y audición.

- Tiempo de seguimiento por un nefrólogo.

- Técnica escogida.

Una vez los pacientes habían iniciado diálisis y se encontraban estables clínicamente, se administraron por personal entrenado los siguientes cuestionarios de escalas psicológicas:

- Cuestionario revisado de personalidad de Eysenck (EPQ-RS), que valora tres dimensiones básicas de la personalidad: neuroticismo, psicoticismo y extraversión, así como una escala de sinceridad. Cada dimensión de la personalidad se considera un continuo, de forma que las personas pueden acercarse a los extremos de la escala en mayor o menor grado. Consta de 48 preguntas que se responden de forma dicotómica, sí o no. Las puntuaciones directas de cada subescala son transformadas en puntuaciones en centiles según los baremos publicados por sexo y edad. El cuestiona- 
rio ha sido adaptado y tipificado en la población española (TEA, ediciones S.A., 1997), siendo uno de los más utilizados en la evaluación de la personalidad (3).

- Escala de ajuste premórbido (Premorbid Adjustment Scale o PAS). Se trata de una escala diseñada para evaluar el nivel de funcionamiento que el individuo ha alcanzado en cada uno de los periodos básicos del desarrollo antes del inicio de la enfermedad. Valora cuatro aspectos: sociabilidad y aislamiento, relación con los compañeros, habilidad para funcionar fuera de la familia nuclear, y facilidad para formar relaciones íntimas. Las puntaciones se convierten en un índice de ajuste, en la que el 0 es la mejor puntuación y el 1, la peor ${ }^{(4)}$.

- Test de afrontamiento (COPE). Es una escala que mide la forma en que los individuos responden cuando se enfrentan a acontecimientos difíciles o estresantes en sus vidas. De los 15 modos de afrontamiento de que consta la escala se han obtenido dos puntuaciones, la adaptativa o la desadaptativa ${ }^{(5)}$.

Para el análisis de los resultados se ha creado una base de datos en Excel y se ha usado el paquete estadístico SPSS para Windows versión 11.5 para realizar la estadística básica, la prueba de $\mathrm{X}^{2}$ para la comparación de proporciones y la prueba t de Student para la comparación de medias. Se han considerado estadísticamente significativas las $p$ inferiores a 0,05.

\section{RESULTADOS}

Se han estudiado un total de 39 pacientes, con una edad media de 63,6 años, predominio de varones y con la nefropatía diabética y vascular como causas más importantes de su IRC. De ellos, 20 pacientes $(51,3 \%)$ optaron por la Hemodiálisis (HD) y 19 (48,7\%) por la Diálisis Peritoneal (DP). Todos los pacientes tenían capacidad para elegir la técnica de diálisis, excepto dos en que sus médicos consideraron que la DP estaba contraindicada por problemas médicos o sociales, en estos dos casos el paciente también prefería realizar HD. En cuatro ocasiones la información sobre las técnicas de diálisis (PAD) se realizó tras la inclusión en programa de diálisis crónica.

Tal y como refleja la tabla 1 , al comparar los pacientes que optaron por la HD y los que eligieron la DP, no hemos hallado diferencias estadísticamente significativas en cuanto a la edad, el sexo, la causa de la IRC, residencia en un ámbito urbano o semirural, estado civil, tipo de convivencia en el domicilio, nivel educativo, proporción de trabajadores activos, categoría laboral cuando eran activos o nivel de ingresos familiares. En ambos grupos, un $80 \%$ de los pacientes fueron seguidos en consultas externas de Nefrología al menos 12 meses antes del inicio de diálisis.

Tabla 1. Características clínicas y sociodemográficas de los pacientes estudiados, comparando los pacientes que eligieron hemodiálisis (HD) y los que eligieron diálisis peritoneal (DP) como técnica de diálisis, tras ser informados mediante el Programa de Ayuda a la Decisión (PAD).

\begin{tabular}{|c|c|c|c|c|}
\hline Variable & $\begin{array}{c}\text { HD } \\
(n=20)\end{array}$ & $\begin{array}{c}\text { DP } \\
(n=19)\end{array}$ & $\begin{array}{c}\text { Total } \\
(n=39)\end{array}$ & $\mathbf{P}$ \\
\hline$\%$ Varones & $75 \%$ & $63,2 \%$ & $69,2 \%$ & NS \\
\hline Edad [media (DE)] & $66,5(10,2)$ & $60,5(15,2)$ & $63,6(13,0)$ & NS \\
\hline $\begin{array}{l}\text { Causa de la IRC } \\
\text { - No filiada } \\
\text { - Glomerular } \\
\text { - Poliquistosis } \\
\text { - Diabetes } \\
\text { - Vascular } \\
\text { - Intersticial } \\
\text { - Otros }\end{array}$ & $\begin{array}{c}10 \% \\
5 \% \\
10 \% \\
30 \% \\
30 \% \\
10 \% \\
5 \%\end{array}$ & $\begin{array}{c}26,3 \% \\
10,5 \% \\
15,8 \% \\
10,5 \% \\
31,6 \% \\
5,3 \% \\
0 \%\end{array}$ & $\begin{array}{c}17,9 \% \\
7,7 \% \\
12,8 \% \\
20,5 \% \\
30,8 \% \\
7,7 \% \\
2,6 \%\end{array}$ & NS \\
\hline $\begin{array}{l}\text { Días desde la realización } \\
\text { del PAD hasta el inicio } \\
\text { de la diálisis [mediana (rango)] }\end{array}$ & $\begin{array}{c}60 \\
\left(-149^{*}, 600\right)\end{array}$ & $\begin{array}{c}205 \\
\left(-61^{*}, 999\right)\end{array}$ & $\begin{array}{c}135 \\
\left(-149^{*}, 999\right)\end{array}$ & NS \\
\hline $\begin{array}{l}\text { Residencia en área rural } \\
\text { o semirural }\end{array}$ & $15 \%$ & $26,3 \%$ & $20,5 \%$ & NS \\
\hline Estado civil (\% casados) & $95 \%$ & $78,9 \%$ & $87,2 \%$ & NS \\
\hline $\begin{array}{l}\text { Convivencia } \\
\text { - Vive solo } \\
\text { - En pareja } \\
\text { - Con hijos }\end{array}$ & $\begin{array}{c}0 \% \\
80 \% \\
20 \%\end{array}$ & $\begin{array}{l}5,3 \% \\
84,2 \% \\
10,5 \%\end{array}$ & $\begin{array}{l}2,6 \% \\
82,1 \% \\
15,4 \%\end{array}$ & NS \\
\hline $\begin{array}{l}\text { Nivel educativo } \\
\text { - Analfabeto } \\
\text { - Estudios primarios } \\
\text { - Estudios secundarios } \\
\text { - Estudios universitarios }\end{array}$ & $\begin{array}{c}18,8 \% \\
62,5 \% \\
12,5 \% \\
6,3 \%\end{array}$ & $\begin{array}{c}5,5 \% \\
62,1 \% \\
27,8 \% \\
5,6 \%\end{array}$ & $\begin{array}{c}11,8 \% \\
61,8 \% \\
20,6 \% \\
5,9 \%\end{array}$ & NS \\
\hline$\%$ Trabajadores activos & $5,9 \%$ & $5,9 \%$ & $5,9 \%$ & NS \\
\hline $\begin{array}{l}\text { Categoría laboral } \\
\text { - Empresario / directivo } \\
\text { - Profesional liberal } \\
\text { - Trabajador por cuenta ajena } \\
\text { - Jubilado / pensionista } \\
\text { - Parado } \\
\text { - No registrada }\end{array}$ & $\begin{array}{c}5 \% \\
5 \% \\
10 \% \\
75 \% \\
0 \% \\
5 \%\end{array}$ & $\begin{array}{c}5,3 \% \\
10,5 \% \\
21,1 \% \\
52,6 \% \\
10,5 \% \\
0 \%\end{array}$ & $\begin{array}{c}5,1 \% \\
7,7 \% \\
15,4 \% \\
64,1 \% \\
5,1 \% \\
2,6 \%\end{array}$ & 0,04 \\
\hline $\begin{array}{l}\text { Nivel de ingresos menor de } \\
12000 € \text { anuales } \\
\text { Problemas de visión } \\
\text { Problemas de audición } \\
\text { Seguimiento nefrológico más } \\
\text { de } 12 \text { meses antes de la diálisis }\end{array}$ & $\begin{array}{c}35,7 \% \\
20 \% \\
5 \% \\
\\
80 \%\end{array}$ & $\begin{array}{c}17,6 \% \\
21 \% \\
5,3 \% \\
84,2 \%\end{array}$ & $\begin{array}{l}25,9 \% \\
20,5 \% \\
10,3 \% \\
\\
82,1 \%\end{array}$ & $\begin{array}{l}\text { NS } \\
\text { NS } \\
\text { NS } \\
\text { NS }\end{array}$ \\
\hline
\end{tabular}

DE: desviación estándar. NS: no significativo ( $p>0,05)$.

"Hubo 4 pacientes en que el PAD se realizó tras el inicio de diálisis. 
Entre la realización del PAD y la entrada en diálisis pasaron una mediana de 135 días (rango: entre 144 días tras la entrada en diálisis hasta 999 días antes de iniciar diálisis), sin que se encontrasen diferencias estadísticamente significativas entre los pacientes que eligieron HD o DP.

Los resultados de los tests psicológicos se resumen en la tabla 2. No hemos encontrado diferencias en cuanto a los antecedentes psiquiátricos personales o familiares, la escala de ajuste premórbido o las tres dimensiones del test de personalidad de Eysenck (extraversión, neuroticismo y psicoticismo). Solamente se ha observado una diferencia estadísticamente significativa en cuanto a la escala desadaptativa del test COPE de afrontamiento, de manera que los enfermos que escogieron DP tenían un mejor nivel de afrontamiento de los problemas.

Tabla 2. Comparación de los resultados de los tests psicológicos entre los pacientes que eligieron hemodiálisis (HD) y los que eligieron diálisis peritoneal (DP) como técnica de diálisis, tras ser informados mediante el Programa de Ayuda a la Decisión (PAD).

\begin{tabular}{|l|c|c|c|c|}
\hline Variable & $\begin{array}{c}\text { HD } \\
\text { (n= 20) }\end{array}$ & $\begin{array}{c}\text { DP } \\
\text { (n= 19) }\end{array}$ & $\begin{array}{c}\text { Total } \\
\text { (n= 39) }\end{array}$ & P \\
\hline \hline $\begin{array}{l}\text { Antecedentes psiquiátricos } \\
\text { familiares }\end{array}$ & $11,8 \%$ & $0 \%$ & $6,9 \%$ & 0,24 \\
\hline $\begin{array}{l}\text { Antecedentes psiquiátricos } \\
\text { personales }\end{array}$ & $15 \%$ & $0 \%$ & $8,1 \%$ & 0,15 \\
\hline $\begin{array}{l}\text { Cuestionario de personalidad } \\
\text { de Eysenck (EPQ-RS) }\end{array}$ & & & & \\
Imedia (DE)]: & $48,6(9,0)$ & $52,9(9,8)$ & $50,7(9,54)$ & 0,16 \\
- Extraversión & $48,6(8,6)$ & $46,2(10,0)$ & $47,4(9,29)$ & 0,41 \\
- Neuroticismo & $46,9(9,2)$ & $48,7(10,0)$ & $47,8(9,53)$ & 0,58 \\
- Psicoticismo & & & & \\
\hline $\begin{array}{l}\text { Escala de ajuste premórbido } \\
\text { (PAS) [media (DE)] }\end{array}$ & $0,16(0,09)$ & $0,12(0,06)$ & $0,14(0,08)$ & 0,15 \\
\hline $\begin{array}{l}\text { Test de afrontamiento (COPE) } \\
\text { Imedia (DE)]: }\end{array}$ & & & & \\
- Escala adaptativa & $2,53(0,32)$ & $2,48(0,47)$ & $2,51(0,40)$ & 0,72 \\
- Escala desadaptativa & $1,63(0,37)$ & $1,40(0,28)$ & $1,52(0,35)$ & 0,036 \\
\hline
\end{tabular}

DE: desviación estándar. NS: no significativo $(p>0,05)$.

\section{DISCUSIÓN}

En nuestro centro la elección del tipo de diálisis se ha realizado siempre después de un programa de información sobre las dos técnicas: la hemodiálisis y la diálisis peritoneal. A pesar que ambas técnicas se consideran en plan de igualdad por los profesionales, sólo de un 20 a un 25\% de los enfermos escogen la DP como primera opción. Probablemente las principales razones para que nuestros pacientes no escojan una téc- nica domiciliaria como la DP, que requiere una cierta autonomía, serían la falta de cultura del autocuidado y que nuestro sistema sanitario dispone de puestos de HD sin límite.

Por otra parte, también hemos observado que un número no despreciable de enfermos se transfiere precozmente de DP a HD por inadaptación a la DP $(17 \%$ en nuestro centro) $)^{(6)}$, que se manifiesta en forma de diversos síntomas de intolerancia física que probablemente traducen una incorrecta elección inicial de la técnica. De los 19 pacientes que hacen DP, 3 pacientes pasaron en pocos meses a HD; estos enfermos no tenían rasgos de personalidad diferentes al resto, aunque no se pueden extraer conclusiones por el escaso número de enfermos.

Nuestra impresión subjetiva es que cuando el paciente participa en la elección de la técnica, múltiples factores individuales se imbrican para tomar la decisión final y que en definitiva, aspectos de la personalidad del enfermo son muy relevantes. El limitado número de pacientes, en este estudio los pacientes con menor puntuación en la escala desadaptativa de afrontamiento, es decir, aquellos que tenían como característica psicológica una mayor capacidad para afrontar acontecimientos difíciles o estresantes (y la necesidad inminente de iniciar tratamiento sustitutivo renal es un buen ejemplo), son los que eligieron preferentemente la DP como técnica de diálisis. Probablemente porque estos pacientes eran también los más predispuestos al autocuidado. Sólo encontramos una tendencia a una mayor extraversión entre los pacientes que optaban por la DP. No hallamos diferencias en otras variables que, sobre el papel, también podrían influir en la decisión como, por ejemplo, rasgos de personalidad como el neuroticismo (que suelen relacionarse con un mayor nivel de ansiedad) o el psicoticismo, la adaptación social, la edad, el sexo, el nivel educativo o de renta o el grado de apoyo familiar y social.

\section{CONCLUSIÓN}

Algunos rasgos psicológicos de la personalidad del individuo medibles mediante tests psicológicos influyen en la elección de la técnica de diálisis.

En nuestro estudio:

1. Los enfermos que escogieron DP tenían un mayor nivel de afrontamiento de los problemas.

2. El tipo de personalidad y el nivel de adaptación social y el soporte psicosocial no influyen en la elección de la técnica. 


\section{BIBLIOGRAFÍA}

1. Breckenridge DM. Decisiones regarding didysin tractment modality: a holistic perspective. Holist Nurs Pract 1997 Oct; 12: 54-61.

2. García M, Rodríguez A, Ponz E, Almirall J, Ramírez J. Opciones ante la insuficiencia renal crónica en un hospital comunitario. Nefrología 1995; 15: 349-355.

3. Ortet G, Ibanez MI, Moro M, et al. Cuestionario de personalidad de Eysenck. Ediciones TEA 1997.

4. Cannon-Spoor HE, Potkin SG, Wyatt RJ. Measurement of premorbid adjusment in chronic schizophrenia. Schizophr Bull 1982; 8:470-84.
5. Cruzado J A, Vázquez C, Crespo M. Escala de afrontamiento COPE.

6. Blasco Cabañas C, Ponz Clemente E, Mañé Buxó N, Martínez Ocaña JC, Marquina Parra D, Yuste Giménez E, García García M. Estudio detallado de las causas de transferencia de diálisis peritoneal a hemodiálisis en un servicio de nefrología. Rev Soc Esp Enferm Nefrol 2004; 7: 43-48.

7. Devins GM, Mendelssohn DC, Barré PE, Binik YM. Predialysis psychoeducational intervention and coping styles influence time to diálisis in chronic kidney disease. Am J Kidney Dis 2003; 42: 693-703. 